\title{
A Comparative Analysis of the Global Electrophilicity Power of Some Cationic Dyes. Understanding Selective Removal Dyes From Mixture Solution
}

\author{
Abdelmajid Regti ${ }^{1,2, *}$, Hicham Ben Elayouchia ${ }^{1}$, Mohammadine El Haddad ${ }^{1}$, My $\operatorname{Rachid~Laamari~}^{1}$ \\ 1 Laboratory of Analytical and Molecular Chemistry (LCAM), Poly-Disciplinary Faculty of Safi, Cadi Ayyad University, \\ BP 4162, 46000 Safi, Morocco \\ 2 Faculty of Applied Sciences, Ait Melloul, Ibn Zohr University, Agadir, Morocco \\ * Correspondence: abdelmajid.regti@gmail.com;
}

Scopus Author ID 57204952778

Received: 29.03.2021; Revised: 29.04.2021; Accepted: 3.05.2021; Published: 9.05.2021

\begin{abstract}
Some bibliographic findings of dyes' adsorption from a mixture state suggest that certain dyes are more likely to be adsorbed first on the adsorbent surface than others, and therefore attracted strongly to the adsorbent surface. To explain the phenomenon, DFT calculations are applied. In this fact, the global electrophilicity index $\omega$ of some cationic dyes has been evaluated. The results show that, for studied electrophilic dyes, the molecule with the greatest global electrophilicity power is favored to be adsorbed on the surface's anionic sites. In addition, local electrophilic Parr and Fukui functions were introduced to characterize the most reactive adsorption sites properly and indicate successfully the same adsorption centers. The success of DFT calculations in explaining and predicting the selective dye was assessed.
\end{abstract}

Keywords: biosorbent; adsorption; DFT calculations; global electrophilicity; local electrophilicity; mixture solution; parr and fukui functions.

(C) 2021 by the authors. This article is an open-access article distributed under the terms and conditions of the Creative Commons Attribution (CC BY) license (https://creativecommons.org/licenses/by/4.0/).

\section{Introduction}

The textile effluent contains a high level of organic pollutants which disturb the natural balance by reducing photosynthetic activity and primary production, and it is toxic. Some of the dyes from textile industries degrade into aromatic amines that are carcinogenic under anaerobic conditions and cause serious health problems in humans and other animals [1]. These substances are often resistant to degradation by biological methods and are not removed effectively by conventional Physico-chemical treatment methods [2]. The removal of it from industrial wastewaters is important in protecting public health, the environment, and aquatic life. The methods employed for removing dyes from contaminated wastewaters are coagulation, flocculation, biological oxidation, solvent extraction, chemical precipitation, reverse osmosis, ion exchange, filtration, and membrane processes, etc. [3]. Most of these technologies have obvious disadvantages, including incomplete dye removal, high reagent and energy requirements, low selectivity, high investment and operating costs, and the generation of secondary waste that is difficult to dispose of. Recently, the possibility of using an adsorption process to remove dyes emitted by the textile industry has been considered. Dye adsorption is a process of transfer of molecule dyes from the bulk solution phase to the surface/interface. The adsorption of dyes at the solid-liquid interface plays an important role in many 
technological and industrial applications. [4,5]. Several researchers are interested in the adsorption of toxic dye mixtures because the textile industry wastewater contains several dyes (bi or multi-adsorption systems) [6,7]. Many unconventional low-cost adsorbents have been used to remove dyes in wastewater[8-12]. Quantum chemical techniques have already been verified to be very beneficial in figuring out the molecular structure and elucidating the electronic structure and reactivity [13-19].

Density functional theory (DFT) has been demonstrated to be a significant instrument in current quantum chemistry given its capacity to incorporate some impacts of electron correlation at an enormously decreased calculation cost. [20,21]. It has also provided a very useful framework for developing new criteria for rationalizing, predicting, and eventually understanding many aspects of chemical processes [22]. Various chemical concepts that are now widely used as descriptors of chemical reactivity, such as electronegativity, hardness, or softness quantities, appear naturally within DFT [23]. The Fukui function calculates the local electron density/population displacements corresponding to a single electron inflow and describes the electron gas's relative local softness [24]. According to several studies, molecular weight is the major factor that affects selective dye adsorption from bi or multi-mixture systems. The major aim of this study is to carry out a DFT calculation on the electronic parameters of some hazardous cationic dyes and to determine a relationship between some quantum chemical parameters obtained from the structure of dyes and the selectivity during simultaneous removal of dyes from the aqueous solution obtained experimentally from previous bibliographic researches. It also proposes a parr and Fukui functions models that would clearly show the local adsorption sites.

\section{Materials and Methods}

\subsection{Selective adsorption of dyes from a multi-dyes mixture.}

When a mixture of substances is in contact with an adsorbent surface, there is competition for the different adsorptive interfaces. The presence of other solutes in the mixture affects the adsorption of the first, the adsorption of some mixtures of dyes was already studied by several researchers, and they indicate the favorability of some dyes in comparison with others [25], some dyes are faster than others, and this might be caused by the large difference between the molecule sizes of dyes. To explain the results, the researchers affirm that it is easier for the molecule with a smaller molecule size to diffuse into the sites of adsorbent than heavy dyes[26,27]. The results of competitive adsorption of some dyes in a mixture solution are illustrated in Table 1. For mixture 1, a competition between two cationic dyes onto anionic sites of adsorbent was assessed (methylene blue (MW: $319.9 \mathrm{~g} / \mathrm{mol}$ ) and malachite green $(\mathrm{MW}=364.91 \mathrm{~g} / \mathrm{mol})$ ), the result indicates that malachite green (heavy dyes) was favored.

Table 1. Result of selective adsorption of dyes from mixture state.

\begin{tabular}{c|c|c|c} 
Mixture number & Mixture dye & Favored Dye & Reference \\
\hline 1 & MB+MG & MG & {$[28]$} \\
\hline 2 & MB+CV & MB & MG \\
\hline 3 & MG+CV & BB41 & {$[30,31]$} \\
\hline 4 & BB41+BY28 & MB & {$[25]$} \\
\hline 5 & MB+BY28 & MB & {$[32]$} \\
\hline 6 & MB+RB & {$[33]$}
\end{tabular}


The difference between the molecular weight of dyes failed to explain this dye's selectivity onto the adsorbent surface. To give a new reason for understanding selective adsorption, we will use the reactivity indices derived from DFT to justify the experimental results.

\subsection{Computational details and calculations.}

The DFT/B3LYP/6-31G (d,p) level of theory, which is implemented in the Gaussian 98 package of programs, was used to compute the results. The molecules' geometry was optimized by the density functional theory (DFT) to create a correlation between experimental data and the investigated dyes' structural and electronic characteristics. The optimized structures and some properties of the investigated dyes are given in Table2. Various parameters were determined and examined, such as Eномо, Elumo, dipole moment $(\mu)$, absolute hardness $(\eta)$, electrophilicity power $(\dot{\omega})$, Nucleophilicity $(N)$, and the total energy (E tot ). The parr and Fukui indices were used to examine local reactivity since they indicate the reactive region in the form of nucleophilic and electrophilic behavior of each atom in the molecule.

Table 2. Optimized structures of dyes using DFT at the B3LYP/6-31G+ (d,p) and some properties of dyes.

\begin{tabular}{|c|c|c|c|}
\hline Dye & Optimized Structure & MW(g/mol) & $\lambda \max (\mathrm{nm})$ \\
\hline Methylene blue & & 319.9 & 664 \\
\hline Malachite green & & 364.91 & 618 \\
\hline Crystal violet & & 407.98 & 580 \\
\hline Basic blue 41 & & 482.57 & 606 \\
\hline
\end{tabular}




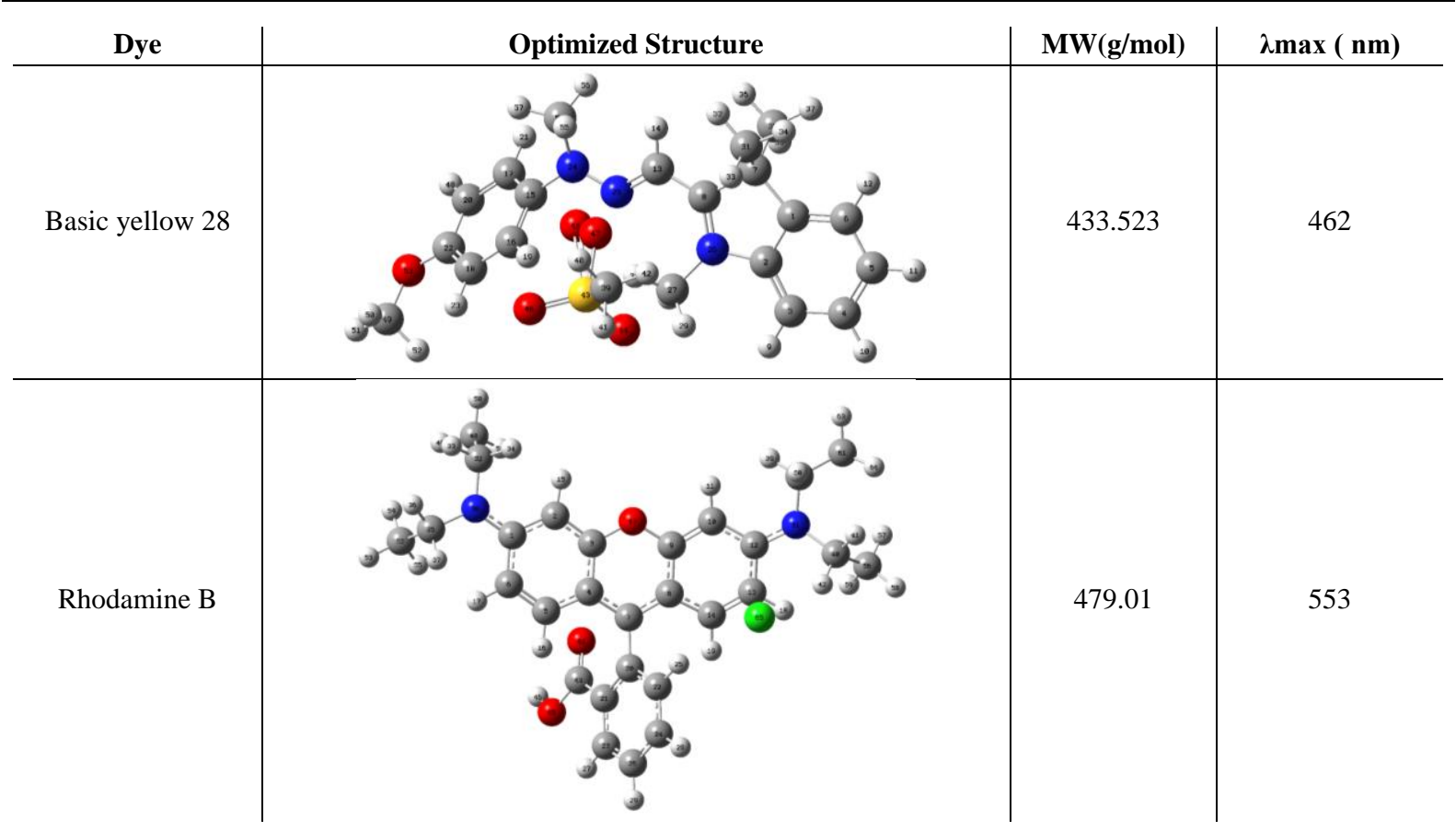

\section{Results and Discussion}

\subsection{Frontier molecular orbital (FMO) level calculations.}

Chemical reactivity is a function of the interaction between the HOMO and LUMO levels of reacting species, according to the frontier molecular orbital theory [22]. The highest occupied molecular orbital energy (Еномо) is a measurement of a molecule's tendency to donate electrons. As a result, higher Еномо values suggest a greater tendency for electron donation. Elumo indicates the molecule's ability to accept electrons, enhancing the adsorption of dyes on anionic sites of the surface. The frontier molecular orbital of the studied compounds is represented in Table 3. However, the LUMO extends over the benzene rings, sulfur, and nitrogen atoms for $\mathrm{BM}$. Benzene rings and nitrogen atoms for $\mathrm{MG}$ and $\mathrm{CV}$, benzene rings for $\mathrm{BB} 41$, benzene rings, and nitro for BY28 for the RB, which extends over the benzene rings. LUMO results indicate all studied dyes' electrophilic character and their ability to be attached to anionic adsorbent sites. The global electrophilicity power difference may explain the competitive and selective adsorption of cationic dyes on anionic adsorbent sites. Great global electrophilicity power generates a great interaction with anionic surface sites. Table 4 shows the calculated energies of the frontier orbitals.

Table 3. The highest occupied molecular orbital (HOMO) molecular orbitals and the lowest unoccupied molecular orbital (LUMO) of the studied dyes using B3LYP/6-31+G(d,p).

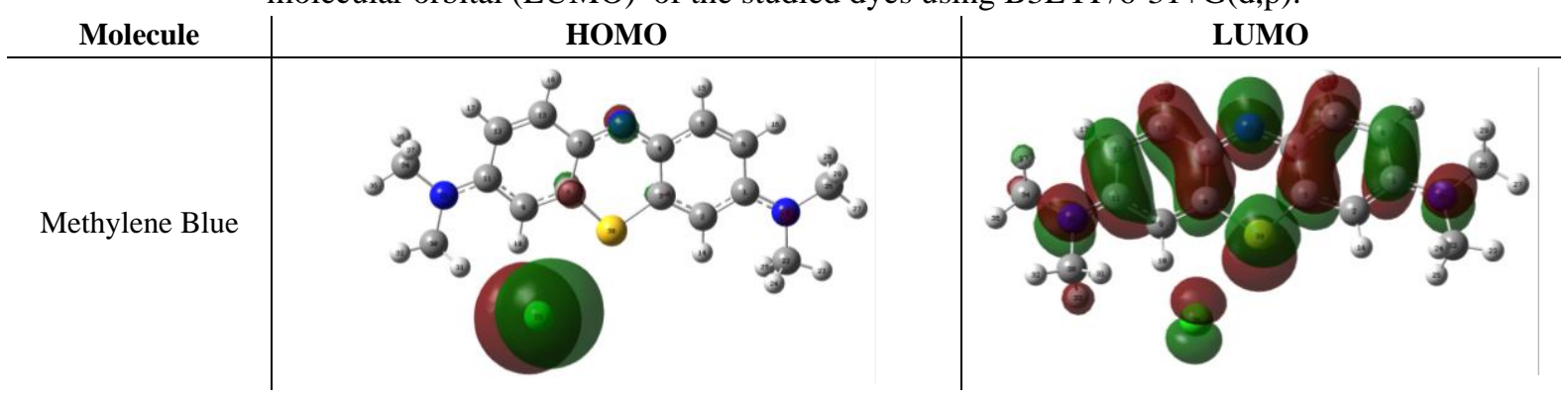




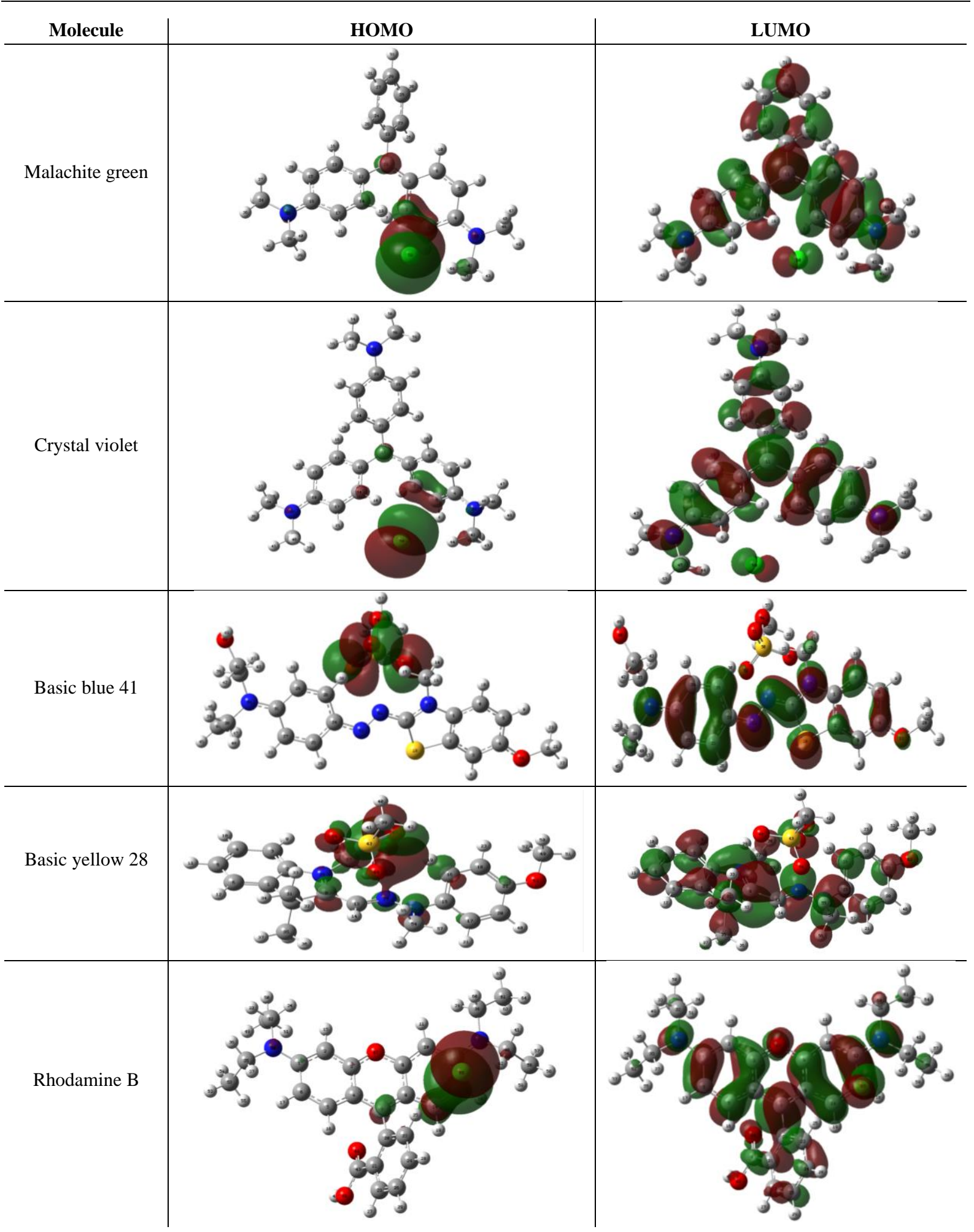

3.2. Global molecular reactivity.

The global indexes defined in the context of the DFT, the electronic chemical potential $(\mu)$, chemical hardness $(\eta)$, and electrophilicity power $(\omega)$, values were approximated in terms of the one-electron energies of the frontier molecular orbital (FMO) HOMO and LUMO, $\mathrm{E}_{\mathrm{H}}$ and $\mathrm{E}_{\mathrm{L}}$ using the expressions:

$$
\mu=\frac{E_{H O M O}+E_{L U M O}}{2}
$$

here $\mu$ is the electronic chemical potential. Hardness $(\eta)$ has been defined within the DFT as: 
$\eta=\left(E_{L}-E_{H}\right)$

Parr [23] have defined the electrophilicity index $(\omega)$ as follows:

$$
\omega=\frac{\mu^{2}}{2 \eta}
$$

L.R. Domingo have been proposed for closed-shell organic molecules an empirical (relative) nucleophilic index N based on the HOMO energies [23], Еномо obtained within the Kohn-Sham scheme [20] and defined as:

$$
N=E_{\text {номо }}(N u)-E_{\text {номо }}(T C E)
$$

Tetracyanoethylene (TCE) is used as a reference in the nucleophilicity scale.

Table 4 displays the global reactivity indices $\mu, \eta$, $\omega$, and $N$ for each dye studied. This index measures the tendency of chemical species to accept electrons, according to the concept. A low value of $\mu, \omega$ is indicative of a good, more reactive nucleophilic, whereas a high value of $\mu, \omega$ is indicative of a good electrophilic. By examining the electrophilicity $\omega$ values for these dyes, it can be noted that, for all mixtures, favored dyes have the greatest value of electrophilicity power $\omega$, indicating that interaction power with anionic sites can explain the selective adsorption successfully. The behavior can be explained in terms of the favored dye's electrophilicity power, making it more reactive and easily reached to adsorbent sites. A similar result was found in previous research [26].

Table 4. Quantum chemical descriptors of the studied dyes calculated at B3LYP/6-31G+(d,p).

\begin{tabular}{l|c|c|c|c|c|c|c|c} 
& $\mathbf{E}_{\mathbf{T}}(\mathbf{u . a})$ & $\mathbf{E}_{\text {Hомо }}(\mathbf{e V})$ & $\mathbf{E}_{\mathbf{L U M O}}(\mathbf{e V})$ & $\boldsymbol{\mu}(\mathbf{D e b y e})$ & $\boldsymbol{\mu}(\mathbf{e V})$ & $\boldsymbol{\eta}(\mathbf{e V})$ & $\boldsymbol{\omega}(\mathbf{e V})$ & $\mathbf{N}(\mathbf{e V})$ \\
\hline MG & -1461.24 & -4.439 & -3.054 & 9.486 & -3.746 & 1.385 & 5.06 & 4.96 \\
\hline MB & -1643.20 & -4.991 & -3.098 & 7.3816 & -4.045 & 1.892 & 4.32 & 4.41 \\
\hline CV & -1595.22 & 4.202 & -2.758 & 14.2227 & -3.48 & 1.443 & 4.19 & 5.20 \\
\hline RB & -1881.027 & -3.889 & -2.501 & 11.7978 & -3.195 & 1.387 & 3.67 & 5.49 \\
\hline BB41 & -2244.47 & -5.522 & -3.328 & 9.2916 & -4.42 & 2.19 & 4.46 & 3.88 \\
\hline BY28 & -1754.90 & -4.727 & -2.46 & 4.0424 & -3.593 & 2.266 & 2.85 & 4.67
\end{tabular}

\subsection{Local selectivity using Parr and Fukui functions.}

Fukui functions compute local reactivity indices that make it possible to rationalize individual molecular orbital contributions' reactivity. The condensed Fukui function and local softness indices allow one to distinguish each part of the molecule based on its distinct chemical behavior due to the different substituted functional groups [34]. The fk+ measures the changes of density when the molecules gain electrons, and it corresponds to reactivity for nucleophilic attack. On the other hand, fk- corresponds to reactivity for electrophilic attack or when the molecule loses electrons. For a system of $\mathrm{N}$ electrons, independent single-point calculations are made, at the same level of theory, for corresponding $\mathrm{N}+1$ and $\mathrm{N}-1$ electron systems. The natural population analysis yields $\mathrm{Pk}(\mathrm{N}-1), \mathrm{Pk}(\mathrm{N})$, and $\mathrm{Pk}(\mathrm{N}+1)$, the population for all $\mathrm{k}$ atoms. The condensed Fukui functions are computed using the finite-difference approximation as follows:

$\mathrm{F}_{\mathrm{k}}^{+}=\left[\mathrm{P}_{\mathrm{K}}(\mathrm{N}+1)-\mathrm{P}_{\mathrm{K}}(\mathrm{N})\right]$
$\mathrm{F}_{\mathrm{k}}^{-}=\left[\mathrm{P}_{\mathrm{K}}(\mathrm{N})-\mathrm{P}_{\mathrm{K}}(\mathrm{N}-1)\right]$
$N_{k}=N f_{k}^{-} \quad$ And $\omega_{k}=\omega f_{k}^{+}$

https://nanobioletters.com/
(For nucleophilic attack)

(For electrophilic attack)

(local indices) 
In Table 5, it is displayed the most relevant values of the natural population $(\mathrm{P}(\mathrm{N}), \mathrm{P}(\mathrm{N}-$ 1 ), and $\mathrm{P}(\mathrm{N}+1)$ with the corresponding values of the Fukui functions ( $f_{\mathrm{k}}-$ and $\mathrm{f}_{\mathrm{k}}+$ ), and the local electrophilicity and nucleophilicity indices $\omega_{\mathrm{k}}$, and $\mathrm{N}_{\mathrm{K}}$ of the studied dyes. The calculated Fukui functions indicate that the C4, C6, C19, and C29 atoms in MG dyes have the highest values of $\omega_{\mathrm{k}}$ which means that they would probably be the favorite sites for nucleophilic attacks, N19 and S38 for MB, C11 for CV, C7, C12 for RB, N12, S18, and C31for BB41, C8, N24 and N25 for BY28.

Table 5. Prediction of reactive sites using Fukui functions.

\begin{tabular}{|c|c|c|c|c|c|c|c|c|}
\hline Dye & $\begin{array}{l}\text { No. of } \\
\text { atoms }\end{array}$ & $\mathbf{P}(\mathbf{N})$ & $\mathbf{P}(\mathbf{N}+\mathbf{1})$ & $\mathbf{P}(\mathrm{N}-1)$ & $f_{k}^{-}$ & $f_{k}^{+}$ & $\dot{\omega}_{\mathrm{k}}$ & $\mathbf{N}_{\mathbf{k}}$ \\
\hline \multirow{19}{*}{ MG } & $\mathrm{C} 1$ & 6.12841 & 6.11641 & 6.09844 & 0.02997 & -0.012 & - & 0.14880105 \\
\hline & $\mathrm{C} 2$ & 6.16218 & 6.19198 & 6.16401 & -0.00183 & 0.0298 & 0.150937 & - \\
\hline & $\mathrm{C} 3$ & 6.24386 & 6.25555 & 6.23321 & 0.01065 & 0.01169 & 0.05920985 & 0.05287725 \\
\hline & $\mathrm{C} 4$ & 5.73255 & 5.79054 & 5.74794 & -0.01539 & 0.05799 & $\mathbf{0 . 2 9 3 7 1 9 3 5}$ & - \\
\hline & $\mathrm{C} 5$ & 6.29512 & 6.31276 & 6.26916 & 0.02596 & 0.01764 & 0.0893466 & 0.1288914 \\
\hline & C6 & 6.16206 & 6.20758 & 6.15559 & 0.00647 & 0.04552 & 0.2305588 & 0.03212355 \\
\hline & $\mathrm{C} 11$ & 6.1418 & 6.02583 & 5.85586 & 0.28594 & -0.11597 & - & 1.4196921 \\
\hline & $\mathrm{C} 12$ & 6.1418 & 6.11277 & 6.11109 & 0.03071 & -0.02903 & - & 0.15247515 \\
\hline & $\mathrm{C} 13$ & 6.17575 & 6.21654 & 6.16393 & 0.01182 & 0.04079 & 0.20660135 & 0.0586863 \\
\hline & C14 & 6.15833 & 6.18882 & 6.16322 & -0.00489 & 0.03049 & 0.15443185 & - \\
\hline & $\mathrm{C} 15$ & 6.30252 & 6.31742 & 6.27522 & 0.0273 & 0.0149 & 0.0754685 & 0.1355445 \\
\hline & $\mathrm{C} 17$ & 6.29124 & 6.29745 & 6.27367 & 0.01757 & 0.00621 & 0.03145365 & 0.08723505 \\
\hline & C19 & 5.77144 & 5.82609 & 5.75629 & 0.01515 & 0.05465 & 0.27680225 & 0.07521975 \\
\hline & $\mathrm{C} 23$ & 6.20767 & 6.23288 & 6.20167 & 0.006 & 0.02521 & 0.12768865 & 0.02979 \\
\hline & $\mathrm{C} 24$ & 6.20445 & 6.23117 & 6.19868 & 0.00577 & 0.02672 & 0.1353368 & 0.02864805 \\
\hline & $\mathrm{C} 25$ & 6.24491 & 6.25536 & 6.23579 & 0.00912 & 0.01045 & 0.05292925 & 0.0452808 \\
\hline & $\mathrm{C} 29$ & 6.22301 & 6.28148 & 6.19547 & 0.02754 & 0.05847 & 0.29615055 & 0.1367361 \\
\hline & N32 & 7.44502 & 7.49028 & 7.38408 & 0.06094 & 0.04526 & 0.2292419 & 0.3025671 \\
\hline & N33 & 7.40542 & 7.4707 & 7.33956 & 0.06586 & 0.06528 & 0.3306432 & 0.3269949 \\
\hline \multirow{12}{*}{ MB } & $\mathrm{C} 1$ & 5.79591 & 5.83448 & 5.77185 & 0.02406 & 0.03857 & 0.1666224 & 0.10617678 \\
\hline & $\mathrm{C} 2$ & 6.29066 & 6.29636 & 6.24769 & 0.04297 & 0.0057 & 0.024624 & 0.18962661 \\
\hline & $\mathrm{C} 3$ & 6.22655 & 6.22834 & 6.21277 & 0.01378 & 0.00179 & 0.0077328 & 0.06081114 \\
\hline & C5 & 6.1731 & 6.22298 & 6.14615 & 0.02695 & 0.04988 & 0.2154816 & 0.11893035 \\
\hline & C6 & 6.27703 & 6.30876 & 6.26674 & 0.01029 & 0.03173 & 0.1370736 & 0.04540977 \\
\hline & C9 & 6.28964 & 6.31115 & 6.24695 & 0.04269 & 0.02151 & 0.0929232 & 0.18839097 \\
\hline & $\mathrm{C} 11$ & 5.79574 & 5.8278 & 5.77179 & 0.02395 & 0.03206 & 0.1384992 & 0.10569135 \\
\hline & $\mathrm{C} 13$ & 6.18491 & 6.22297 & 6.15795 & 0.02696 & 0.03806 & 0.1644192 & 0.11897448 \\
\hline & N19 & 7.39226 & 7.50745 & 7.32725 & 0.06501 & 0.11519 & 0.4976208 & 0.28688913 \\
\hline & $\mathrm{N} 20$ & 7.43811 & 7.49313 & 7.37644 & 0.06167 & 0.05502 & 0.2376864 & 0.27214971 \\
\hline & N21 & 7.4377 & 7.49257 & 7.37605 & 0.06165 & 0.05487 & 0.2370384 & 0.27206145 \\
\hline & S38 & 15.27944 & 15.39726 & 15.30389 & -0.02445 & 0.11782 & 0.5089824 & - \\
\hline \multirow{17}{*}{$\mathrm{CV}$} & $\mathrm{C} 2$ & 6.1626 & 6.19274 & 6.17025 & -0.00765 & 0.03014 & 0.1262866 & - \\
\hline & $\mathrm{C} 3$ & 6.24646 & 6.2574 & 6.23054 & 0.01592 & 0.01094 & 0.0458386 & 0.082784 \\
\hline & $\mathrm{C} 4$ & 5.73958 & 5.79519 & 5.75839 & -0.01881 & 0.05561 & 0.2330059 & - \\
\hline & $\mathrm{C} 5$ & 6.2979 & 6.31295 & 6.2687 & 0.0292 & 0.01505 & 0.0630595 & 0.15184 \\
\hline & C6 & 6.1671 & 6.21003 & 6.16392 & 0.00318 & 0.04293 & 0.1798767 & 0.016536 \\
\hline & $\mathrm{C} 11$ & 5.86824 & 6.02381 & 5.86932 & -0.00108 & 0.15557 & $\mathbf{0 . 6 5 1 8 3 8 3}$ & - \\
\hline & $\mathrm{C} 13$ & 6.17987 & 6.21984 & 6.17036 & 0.00951 & 0.03997 & 0.1674743 & 0.049452 \\
\hline & $\mathrm{C} 14$ & 6.14485 & 6.17728 & 6.1539 & -0.00905 & 0.03243 & 0.1358817 & - \\
\hline & $\mathrm{C} 15$ & 6.30619 & 6.31864 & 6.2833 & 0.02289 & 0.01245 & 0.0521655 & 0.119028 \\
\hline & $\mathrm{C} 17$ & 6.29774 & 6.30302 & 6.28399 & 0.01375 & 0.00528 & 0.0221232 & 0.0715 \\
\hline & $\mathrm{C} 19$ & 5.7751 & 5.82905 & 5.76211 & 0.01299 & 0.05395 & 0.2260505 & 0.067548 \\
\hline & $\mathrm{C} 23$ & 6.17957 & 6.20927 & 6.17812 & 0.00145 & 0.0297 & 0.124443 & 0.00754 \\
\hline & $\mathrm{C} 24$ & 6.17773 & 6.20831 & 6.17424 & 0.00349 & 0.03058 & 0.1281302 & 0.018148 \\
\hline & $\mathrm{C} 29$ & 5.79004 & 5.84047 & 5.77307 & 0.01697 & 0.05043 & 0.2113017 & 0.088244 \\
\hline & N32 & 7.45082 & 7.49263 & 7.40375 & 0.04707 & 0.04181 & 0.1751839 & 0.244764 \\
\hline & N33 & 7.41776 & 7.47689 & 7.34383 & 0.07393 & 0.05913 & 0.2477547 & 0.384436 \\
\hline & N51 & 7.46685 & 7.49844 & 7.4319 & 0.03495 & 0.03159 & 0.1323621 & 0.18174 \\
\hline \multirow{4}{*}{$\mathrm{RB}$} & $\mathrm{C} 1$ & 5.78054 & 5.82185 & 5.75669 & 0.02385 & 0.04131 & 0.1516077 & 0.1309365 \\
\hline & C3 & 5.61815 & 5.63637 & 5.60968 & 0.00847 & 0.01822 & 0.0668674 & 0.0465003 \\
\hline & C5 & 6.1744 & 6.19646 & 6.1574 & 0.017 & 0.02206 & 0.0809602 & 0.09333 \\
\hline & C7 & 5.88388 & 6.02362 & 5.83562 & 0.04826 & 0.13974 & 0.5128458 & 0.2649474 \\
\hline
\end{tabular}




\begin{tabular}{|c|c|c|c|c|c|c|c|c|}
\hline Dye & $\begin{array}{l}\text { No. of } \\
\text { atoms }\end{array}$ & $\mathbf{P}(\mathbf{N})$ & $\mathbf{P}(\mathbf{N}+\mathbf{1})$ & $\mathbf{P}(\mathrm{N}-1)$ & $f_{k}^{-}$ & $f_{k}^{+}$ & $\dot{\omega}_{\mathrm{k}}$ & $\mathbf{N}_{\mathbf{k}}$ \\
\hline & C9 & 5.60695 & 5.63946 & 5.597 & 0.00995 & 0.03251 & 0.1193117 & 0.0546255 \\
\hline & C12 & 5.70142 & 5.76681 & 5.72671 & -0.02529 & 0.06539 & 0.2399813 & - \\
\hline & C13 & 6.22229 & 6.24747 & 6.22689 & -0.0046 & 0.02518 & 0.0924106 & - \\
\hline & C14 & 6.16551 & 6.1927 & 6.14146 & 0.02405 & 0.02719 & 0.0997873 & 0.1320345 \\
\hline & N31 & 7.41824 & 7.47556 & 7.36116 & 0.05708 & 0.05732 & 0.2103644 & - \\
\hline \multirow{11}{*}{ BB41 } & $\mathrm{C} 4$ & 5.67011 & 5.70565 & 5.63496 & 0.03515 & 0.03554 & 0.1585084 & 0.136382 \\
\hline & C6 & 6.22381 & 6.25885 & 6.21182 & 0.01199 & 0.03504 & 0.1562784 & 0.0465212 \\
\hline & $\mathrm{C} 7$ & 5.7032 & 5.74259 & 5.7273 & -0.0241 & 0.03939 & 0.1756794 & - \\
\hline & N11 & 7.29677 & 7.33796 & 7.24202 & 0.05475 & 0.04119 & 0.1837074 & 0.21243 \\
\hline & N12 & 7.15086 & 7.28065 & 7.16194 & -0.01108 & 0.12979 & 0.5788634 & - \\
\hline & N17 & 7.33824 & 7.40079 & 7.30617 & 0.03207 & 0.06255 & 0.278973 & 0.1244316 \\
\hline & S18 & 15.50761 & 15.58704 & 15.4607 & 0.04691 & 0.07943 & 0.3542578 & 0.1820108 \\
\hline & $\mathrm{C} 25$ & 6.15507 & 6.20996 & 6.1604 & -0.00533 & 0.05489 & 0.2448094 & - \\
\hline & $\mathrm{C} 26$ & 6.14093 & 6.19699 & 6.15774 & -0.01681 & 0.05606 & 0.2500276 & - \\
\hline & C31 & 5.7432 & 5.82246 & 5.74248 & 0.00072 & 0.07926 & 0.3534996 & 0.0027936 \\
\hline & N34 & 7.42474 & 7.4849 & 7.3175 & 0.10724 & 0.06016 & 0.2683136 & 0.4160912 \\
\hline \multirow{10}{*}{ BY28 } & $\mathrm{C} 1$ & 6.02853 & 6.04379 & 6.02019 & 0.00834 & 0.01526 & 0.043491 & 0.03900618 \\
\hline & $\mathrm{C} 3$ & 6.25396 & 6.29156 & 6.24241 & 0.01155 & 0.0376 & 0.10716 & 0.05401935 \\
\hline & $\mathrm{C} 5$ & 6.24495 & 6.30563 & 6.21174 & 0.03321 & 0.06068 & 0.172938 & 0.15532317 \\
\hline & $\mathrm{C} 8$ & 5.65693 & 5.81048 & 5.65938 & -0.00245 & 0.15355 & 0.4376175 & - \\
\hline & $\mathrm{C} 16$ & 6.2111 & 6.22294 & 6.20239 & 0.00871 & 0.01184 & 0.033744 & 0.04073667 \\
\hline & C17 & 6.24259 & 6.26137 & 6.23023 & 0.01236 & 0.01878 & 0.053523 & 0.05780772 \\
\hline & $\mathrm{C} 22$ & 5.68042 & 5.71925 & 5.64718 & 0.03324 & 0.03883 & 0.1106655 & 0.15546348 \\
\hline & N24 & 7.16372 & 7.25386 & 7.12553 & 0.03819 & 0.09014 & 0.256899 & 0.17861463 \\
\hline & $\mathrm{N} 25$ & 7.12188 & 7.25401 & 7.15164 & -0.02976 & 0.13213 & 0.3765705 & - \\
\hline & N26 & 7.33418 & 7.39626 & 7.30895 & 0.02523 & 0.06208 & 0.176928 & 0.11800071 \\
\hline
\end{tabular}

The local electrophilic, $\mathrm{Pk}+$, and nucleophilic, $\mathrm{Pk}$ - Parr functions obtained from the Mulliken atomic spin density (ASD) study at the radical cation and radical anion of the corresponding reagents have been reported using electron localization function analysis. These local functions allow the identification of a molecule's most electrophilic and nucleophilic centers. The local electrophilicity $\omega_{\mathrm{k}}$ and local nucleophilicity $\mathrm{N}_{\mathrm{K}}$ indices can be redefined as follows[34]:

$$
\begin{aligned}
& \omega_{k}=\omega P_{k}^{+} \\
& N_{k}=N P_{k}^{-}
\end{aligned}
$$

Where $\omega$ and $\mathrm{N}$ are the global electrophilicity and nucleophilicity index. In table 6 , it is displayed the local nucleophilic $P_{k}^{-}$Parr functions of the cationic system and electrophilic $P_{k}^{+}$ Parr functions of the anionic system, and the local electrophilicity and nucleophilicity of the studied dyes. On the other hand, analysis of the electrophilic $\mathrm{P}_{\mathrm{k}}^{+}$Parr functions and their derivative local electrophilic $\omega \mathrm{k}$ of the studied dyes indicate that the $\mathrm{C} 4, \mathrm{C} 6, \mathrm{C} 19$, and $\mathrm{C} 29$ carbons are the most electrophilic sites in MG dyes, which means that it would probably be the favorite sites for nucleophilic attacks, N19 and S38 for MB, C11 for CV, C7 and C12 for RB, N12, S18, and C31for BB41 and C8, N24 and N25 for BY28. The calculated Parr and Fukui functions are in good agreement indicating practically the same electrophilic centers.

Table 5. Prediction of reactive sites using Parr functions

\begin{tabular}{c|c|c|c|c|c}
\multicolumn{1}{c}{ Dye } & No. of atoms & $\boldsymbol{P}_{\boldsymbol{k}}^{+}$ & $\boldsymbol{P}_{\boldsymbol{k}}^{-}$ & $\boldsymbol{\omega}_{\mathbf{k}}$ & $\mathbf{N}_{\mathbf{k}}$ \\
\hline \multirow{4}{*}{ MG } & $\mathrm{C} 1$ & - & 0.098037 & - & 0.48675371 \\
\cline { 2 - 6 } & $\mathrm{C} 2$ & 0.108266 & - & 0.54836729 & - \\
\cline { 2 - 6 } & $\mathrm{C} 3$ & - & 0.185658 & & $\mathbf{0 . 9 2 1 7 9 1 9 7}$ \\
\cline { 2 - 6 } & $\mathrm{C} 4$ & 0.145436 & - & $\mathbf{0 . 7 3 6 6 3 3 3 4}$ & - \\
\cline { 2 - 6 } & $\mathrm{C} 5$ & & 0.043037 & - & 0.21367871
\end{tabular}


https://doi.org/10.33263/LIANBS104.29332944

\begin{tabular}{|c|c|c|c|c|c|}
\hline Dye & No. of atoms & $P_{k}^{+}$ & $P_{k}^{-}$ & $\dot{\omega}_{\mathrm{k}}$ & $\mathbf{N}_{\mathbf{k}}$ \\
\hline & C6 & 0.139386 & & 0.70599009 & - \\
\hline & $\mathrm{C} 11$ & 0.533875 & & 2.70407688 & - \\
\hline & $\mathrm{C} 12$ & - & 0.071881 & - & 0.35688917 \\
\hline & $\mathrm{C} 13$ & 0.127001 & - & 0.64326007 & - \\
\hline & $\mathrm{C} 14$ & 0.10764 & - & 0.5451966 & - \\
\hline & $\mathrm{C} 15$ & - & 0.042466 & - & 0.21084369 \\
\hline & $\mathrm{C} 17$ & - & 0.059611 & - & 0.29596862 \\
\hline & C19 & 0.132489 & 0.012208 & 0.67105679 & 0.06061272 \\
\hline & $\mathrm{C} 22$ & - & 0.02784 & - & 0.1382256 \\
\hline & $\mathrm{C} 23$ & 0.10764 & - & 0.5451966 & - \\
\hline & $\mathrm{C} 24$ & 0.112362 & - & 0.56911353 & - \\
\hline & $\mathrm{C} 25$ & - & 0.009082 & - & 0.04509213 \\
\hline & $\mathrm{C} 29$ & 0.125561 & - & 0.63596647 & - \\
\hline & N32 & 0.028045 & 0.093169 & 0.14204793 & 0.46258409 \\
\hline & N33 & 0.053225 & 0.185391 & 0.26958463 & 0.92046632 \\
\hline \multirow{12}{*}{$\mathrm{MB}$} & $\mathrm{C} 1$ & 0.101432 & - & 0.43818624 & - \\
\hline & $\mathrm{C} 2$ & - & 0.123984 & - & 0.54714139 \\
\hline & $\mathrm{C} 3$ & 0.102378 & - & 0.44227296 & - \\
\hline & $\mathrm{C} 5$ & 0.124779 & 0.013382 & 0.53904528 & 0.05905477 \\
\hline & $\mathrm{C} 8$ & 0.101875 & - & 0.4401 & - \\
\hline & C9 & - & 0.123872 & - & 0.54664714 \\
\hline & $\mathrm{C} 11$ & 0.102063 & - & 0.44091216 & - \\
\hline & $\mathrm{C} 13$ & 0.124678 & 0.013292 & 0.53860896 & 0.0586576 \\
\hline & N19 & 0.307668 & - & 1.32912576 & - \\
\hline & N20 & 0.026997 & 0.04724 & 0.11662704 & 0.20847012 \\
\hline & N21 & 0.027234 & 0.047669 & 0.11765088 & 0.2103633 \\
\hline & S38 & 0.162535 & 0.124264 & $\mathbf{0 . 7 0 2 1 5 1 2}$ & 0.54837703 \\
\hline \multirow{17}{*}{$\mathrm{CV}$} & $\mathrm{C} 2$ & 0.110395 & - & 0.46255505 & - \\
\hline & $\mathrm{C} 3$ & - & 0.174151 & - & 0.9055852 \\
\hline & $\mathrm{C} 4$ & 0.136793 & - & 0.57316267 & - \\
\hline & $\mathrm{C} 5$ & - & 0.046648 & - & 0.2425696 \\
\hline & C6 & 0.138673 & - & 0.58103987 & - \\
\hline & $\mathrm{C} 11$ & 0.543011 & - & 2.27521609 & - \\
\hline & $\mathrm{C} 13$ & 0.128255 & - & 0.53738845 & - \\
\hline & $\mathrm{C} 14$ & 0.109373 & - & 0.45827287 & - \\
\hline & $\mathrm{C} 15$ & - & 0.024705 & - & 0.128466 \\
\hline & $\mathrm{C} 17$ & - & 0.04847 & - & 0.252044 \\
\hline & C19 & 0.132718 & 0.011408 & 0.55608842 & 0.0593216 \\
\hline & $\mathrm{C} 23$ & 0.113594 & - & 0.47595886 & - \\
\hline & $\mathrm{C} 24$ & 0.108937 & - & 0.45644603 & - \\
\hline & $\mathrm{C} 29$ & 0.114557 & - & 0.47999383 & - \\
\hline & N32 & 0.026434 & 0.060512 & 0.11075846 & 0.3146624 \\
\hline & N33 & 0.045688 & 0.205139 & 0.19143272 & 1.0667228 \\
\hline & N51 & 0.018058 & - & 0.07566302 & - \\
\hline \multirow{10}{*}{$\mathrm{RB}$} & $\mathrm{C} 1$ & 0.136727 & - & 0.50178809 & - \\
\hline & $\mathrm{C} 3$ & 0.097854 & - & 0.35912418 & - \\
\hline & $\mathrm{C} 5$ & 0.132614 & - & 0.48669338 & - \\
\hline & $\mathrm{C} 7$ & 0.405379 & - & 1.48774093 & - \\
\hline & C9 & 0.135318 & - & 0.49661706 & - \\
\hline & $\mathrm{C} 12$ & 0.169622 & - & 0.62251274 & - \\
\hline & C13 & - & 0.149344 & - & 0.81989856 \\
\hline & $\mathrm{C} 14$ & 0.090347 & - & 0.33157349 & - \\
\hline & N31 & - & 0.09147 & - & 0.5021703 \\
\hline & N32 & - & 0.148222 & - & 0.81373878 \\
\hline \multirow{4}{*}{ BB41 } & $\mathrm{C} 4$ & 0.030293 & 0.05646 & 0.13510678 & 0.2190648 \\
\hline & C6 & 0.057454 & - & 0.25624484 & - \\
\hline & $\mathrm{C} 7$ & 0.185392 & - & 0.82684832 & - \\
\hline & N11 & - & 0.141336 & - & 0.54838368 \\
\hline
\end{tabular}




\begin{tabular}{|c|c|c|c|c|c|}
\hline Dye & No. of atoms & $P_{k}^{+}$ & $P_{k}^{-}$ & $\dot{\omega}_{\mathrm{k}}$ & $\mathbf{N}_{\mathbf{k}}$ \\
\hline & N12 & 0.448193 & - & 1.99894078 & - \\
\hline & N17 & 0.07116 & 0.067789 & 0.3173736 & 0.26302132 \\
\hline & S18 & 0.042051 & - & 0.18754746 & - \\
\hline & $\mathrm{C} 25$ & 0.182885 & - & 0.8156671 & - \\
\hline & $\mathrm{C} 26$ & 0.211302 & - & 0.94240692 & - \\
\hline & $\mathrm{C} 31$ & 0.184216 & - & 0.82160336 & - \\
\hline & N34 & 0.05098 & 0.22986 & 0.2273708 & 0.8918568 \\
\hline \multirow{9}{*}{ BY28 } & $\mathrm{C} 1$ & 0.055263 & 0.016058 & 0.15749955 & 0.07510327 \\
\hline & $\mathrm{C} 3$ & 0.052273 & 0.012068 & 0.14897805 & 0.05644204 \\
\hline & $\mathrm{C} 5$ & 0.079232 & 0.024931 & 0.2258112 & 0.11660229 \\
\hline & $\mathrm{C} 8$ & 0.485764 & - & 1.3844274 & - \\
\hline & $\mathrm{C} 16$ & 0.039313 & 0.046455 & 0.11204205 & 0.21727004 \\
\hline & $\mathrm{C} 17$ & 0.033834 & 0.00557 & 0.0964269 & 0.02605089 \\
\hline & $\mathrm{C} 22$ & 0.037078 & 0.052517 & 0.1056723 & 0.24562201 \\
\hline & $\mathrm{N} 24$ & 0.132428 & 0.106262 & 0.3774198 & 0.49698737 \\
\hline & $\mathrm{N} 25$ & 0.36051 & - & 1.0274535 & - \\
\hline
\end{tabular}

\section{Conclusion}

In conclusion, the difference in global electrophilicity power is useful in understanding certain cationic dyes' preferences over multi-mixture dyes; the molecule with the highest electrophilicity value has priority in being adsorbed on anionic sites of the adsorbent. Adsorption is related to electrophilicity power, as great electrophilicity value enhances speed molecule to the adsorbent interface. The local analysis using Parr and Fukui functions shows the centers with the highest value of $\omega_{\mathrm{k}}$, which means that they would probably be the favorite sites for nucleophilic attacks (adsorption sites).

\section{Funding}

This research received no external funding.

\section{Acknowledgments}

One of the authors extends their appreciation to the Moroccan Association of theoretical chemists (AMCT) to access the computational facility.

\section{Conflicts of Interest}

The authors declare no conflict of interest.

\section{References}

1. Anuar, F.I.; Hadibarata, T.; Syafrudin, M.; Fona, Z. Removal of Procion Red MX- 5B from aqueous solution by adsorption on Parkia speciosa (stink bean) peel powder. Biointerface Res. Appl. Chem. 2020, 10, 47744779. https://doi.org/10.33263/BRIAC101.774779.

2. Massoud, K.; Mojtaba, S.; Sahar, M. Removal of Dyes from the Environment by Adsorption Process. Chem. Mater. Eng 2018, 6, 31-35. https://doi.org/10.13189/cme.2018.060201.

3. Vilar, V.J.P.; Botelho, C.M.S.; Boaventura, R.A.R. Methylene blue adsorption by algal biomass based materials: biosorbents characterization and process behavior. J. Hazard. Mater 2007, 147, 120-132. https://doi.org/10.1016/j.jhazmat.2006.12.055.

4. Achary, P.G.R.; Ghosh, M.R.; Mishra, S.P. Insights into the modeling and application of some low cost adsorbents towards $\mathrm{Cr}(\mathrm{VI})$ adsorption. Mater. Today 2020, 30, 267-273. https://doi.org/10.1016/j.matpr.2020.01.433. 
5. Nadir, I.; Achour, Y.; El Kassimi, A.; El Himri, M.; Laamari, M.R.; El Haddad, M. Removal of Antibiotic Sulfamethazine from Aqueous Media. Phys. Chem. Res 2021, 9, 165-180. https://doi.org/10.22036/pcr.2020.249992.1839.

6. El Kassimi, A.; Boutouil, A.; El Himri, M.; Rachid Laamari, M.; El Haddad, M. Selective and competitive removal of three basic dyes from single, binary and ternary systems in aqueous solutions: A combined experimental and theoretical study. J. Saudi Chem. Soc. 2020, 24, 527-544, https://doi.org/10.1016/j.jscs.2020.05.005.

7. Regti, A.; El Kassimi, A.; Laamari, M.R.; El Haddad, M. Competitive adsorption and optimization of binary mixture of textile dyes: A factorial design analysis. J. Assoc. Arab Univ. Basic Appl. Sci. 2017, 24, 1-9. https://doi.org/10.1016/j.jaubas.2016.07.005.

8. Al Farraj, D.A.; Hadibarata, T.; Yuniarto, A.; Syafiuddin, A.; Surtikanti, H.K.; Elshikh, M.S.; Al Khulaifi, M.M.; Al-Kufaidy, R. Characterization of pyrene and chrysene degradation by halophilic Hortaea sp. B15. Bioproc Biosyst Eng 2019, 42, 963-969, https://doi.org/10.1007/s00449-019-02096-8.

9. Ghaedi, M. ; Hassanzadeh, A. ; Kokhdan, S. N. Multiwalled Carbon Nanotubes as Adsorbents for the Kinetic and Equilibrium Study of the Removal of Alizarin Red S and Morin. J. Chem. Eng. Data 2011, 56, 2511-2520. https://doi.org/10.1021/je2000414.

10. El Kassimi, A.; Achour, Y.; El Himri, M.; Laamari, M. R.; El Haddad, M. Optimization of preparation conditions of highly efficient activated carbon for use in water treatment-experimental design approach. Int. J. Environ. Anal. Chem 2021, 1-23. https://doi.org/10.1080/03067319.2020.1861261.

11. Regti, A.; Laamari, M.R.; Stiriba, S.-E.; El Haddad, M. Use of response factorial design for process optimization of basic dye adsorption onto activated carbon derived from Persea species. Microchem. J. 2017, 130, 129-136, https://doi.org/10.1016/j.microc.2016.08.012.

12. Somsesta, N.; Sricharoenchaikul, V.; Aht-Ong, D. Adsorption removal of methylene blue onto activated carbon/cellulose biocomposite films: Equilibrium and kinetic studies. Mater. Chem. Phys 2020, 240. https://doi.org/10.1016/j.matchemphys.2019.122221.

13. Benallou, A. ; Lakbaibi,Z. ; Garmes,H. ; El Abdallaoui, H.E.A. The role of the polarity on the mechanism and selectivity in the [3+2] cycloaddition reaction between CF3-ynone ylide and azide group : A quantum chemical investigation, J. Fluor. Chem 2019, 219 ,79-91. https://doi.org/10.1016/j.jfluchem.2018.12.008.

14. Lakbaibi, Z.; Abou El Makarim, H.; Tabyaoui, M.; EL Hajbi, A. Study of the solvent effects on the formation of $\alpha$-bromoglycidic esters in aliphatic series using the quantum DFT method with B3LYP/6-311G (d, p). $J$. Mater. Environ. Sci 2016, 8, 99-115. http://www.jmaterenvironsci.com/.

15. Jaafar, A.; Ben El Ayouchia, H.; Lakbaibi, Z.; Boussaoud, A.; Jodeh, S.; Azzaoui, K.; Tabyaoui, M. Degradation of Pollutant Dye in Aqueous Solution using Fenton Reaction: A DFT Study. G P Globalize Research Journal of Chemistry 2019, 3, 53-61.

16. Manssouri, M.; Lakbaibi, Z.; Znini, M. Impact of Aaronsohnia pubescens Essential Oil to Prevent Against the Corrosion of Mild Steel in $1.0 \mathrm{M} \mathrm{HCl}$ : Experimental and Computational Modeling Studies. J. Fail. Anal. Prev 2020, 20, 1939-1953. https://doi.org/10.1007/s11668-020-01003-8.

17. Lakbaibi, Z.; Jaafar, A.; Ben El Ayouchia, H.; Tabyaoui, M.; Boussaoud, A. Reactivity and mechanism of nucleophilic addition reaction of amine with alkene: A systematic DFT study. Mediterr. J. Chem 2019, 8, 2529. http://dx.doi.org/10.13171/mjc811902924zlhbea.

18. Ansari, A.; Mounir, M.; Mohamed, Z.; Zouhair ,L.; Mohamed, A. Experimental and theoretical study on corrosion inhibition of new synthesized menthone derivatives (Menthopyrazole compounds) for mild steel in $1 \mathrm{M} \mathrm{HCl}$ solution. Mediterr J Chem 2020, 10, 62-76. https://doi.org/10.13171/mjc101020291189aa.

19. Manssouri, M.; Znini, M.; Lakbaibi, Z. Experimental and computational studies of peril aldehyde isolated from Ammodaucus leucotrichus essential oil as a green corrosion inhibitor for mild steel in $1.0 \mathrm{M} \mathrm{HCl}$. Chem. Pap 2020, 75, 1103-1114. https://doi.org/10.1007/s11696-020-01353-5.

20. Kohn ,W.; L.G,Sham. Self- Consistent equations including exchange and correlation Effects. Phys. Rev 1965, 140, 1133-1138.

21. Fukui, K. The role frontier orbitals in chemical reactions. Angewandte Chemie International Edition in English 1982, 21, 801-876. https://doi.org/10.1002/anie.198208013.

22. Domingo, L.R.; Chamorro, E.; Pérez, P. Understanding the reactivity of captodative ethylenes in polar cycloaddition reactions: A theoretical study. J. Org. Chem 2008, 73, 4615-4624. https://doi.org/10.1021/jo800572a. 
23. Domingo, L.R.; Pérez, P.; Sáez, J. A. Understanding the local reactivity in polar organic reactions through electrophilic and nucleophilic Parr functions. RSC Adv 2013, 3, 1486-1494. https://doi.org/10.1039/C2RA22886F.

24. Domingo, L.R.; Aurell, M.J.; Perez, P.; Saez, J.A. Understanding the origin of the asynchronicity in bondformation in polar cycloaddition reactions. A DFT study of the 1,3-dipolar cycloaddition reaction of carbonyl ylides with 1,2-benzoquinones. RSC Adv 2012, 2, 1334-1342. https://doi.org/10.1039/C1RA00717C.

25. Mazaheri, H.; Ghaedi, M.; Asfaram, A.; Hajati, S. Performance of CuS nanoparticle loaded on activated carbon in the adsorption of methylene blue and bromophenol blue dyes in binary aqueous solutions: Using ultrasound power and optimization by central composite design. J. Mol. Liq. 2016, 219, 667-676, https://doi.org/10.1016/j.molliq.2016.03.050.

26. El Kassimi, A.; Boutouil, A.; El Himri, M.; Laamari, M.; El Haddad, M. Selective and competitive removal of three basic dyes from single, binary and ternary systems in aqueous solutions: A combined experimental and theoretical study. J. Saudi Chem. Soc 2020, 24, 527-544. https://doi.org/10.1016/j.jscs.2020.05.005.

27. Achour, Y.; Khouili, M.; Abderrafia, H.; Melliani, S.; Laamari, M.R.; El Haddad, M. DFT investigations and experimental studies for competitive and adsorptive removal of two cationic dyes onto an eco-friendly material from aqueous media. I. J. E. R 2018, 12, 789-802. https://doi.org/10.1007/s41742-018-0131-x.

28. Deokar, R.; Sabale, A. Biosorption of Methylene Blue and Malachite Green from Binary Solution onto Ulva lactuca. Int. J. Curr. Microbiol. App. Sci 2014, 3, 295-304. http://www.ijcmas.com/.

29. Mahamadi, C.; Mawere, E. Kinetic Modeling of Methylene Blue and Crystal Violet Dyes Adsorption on Alginate-Fixed Water Hyacinth in Single and Binary Systems. Am. J. Analyt Chem 2013, 4, 17-24. https://doi.org/10.4236/ajac.2013.410A3003.

30. Gandhimathi, R.; Srikrishnaperumal, S. ; Ramesh, T.; Sindhu, V.; Veetil Nidheesh, P. Bottom ash adsorption of basic dyes from their binary aqueous solutions. J. Sci. Technol 2013, 35, 339-347.

31. Gandhimathi, R.; Ramesh, S.T.; Sindhu, V; Nidheesh, P.V. Single and Tertiary System Dye Removal from Aqueous Solution Using Bottom Ash: Kinetic and Isotherm Studies. Iran. J. Energy. Environ 2012, 3, 35-45. https://doi.org/10.5829/idosi.ijee.2012.03.01.0113.

32. Regti, A.; Ben El Ayouchia, H.; Laamari, M.R.; Anane, H.; Stiriba, S.E.; EL Haddad, M. Experimental and theoretical study using DFT method for the competitive adsorption of two cationic dyes from wastewaters. Appl. Surf. Sci 2016, 390, 311-319. https://doi.org/10.1016/j.apsusc.2016.08.059.

33. Farhade, J.Z.; Habibi-yangjeh, A. Competitive Adsorption of Methylene Blue and Rhodamine B on Natural Zeolite: Thermodynamic and Kinetic Studies. Chin. J. Chem 2010, 28, 349-356. https://doi.org/10.1002/cjoc.201090078.

34. Wang, A.X.; YU, S.; Wang, X.; Ai, Y.; Tan, X.; Hayat, T; Hu, W. Experimental and Theoretical Study on Competitive Adsorption of Aromatic Compounds on Reduced Graphene Oxides. Mater. Chem. A 2016, 4, 5654-5662. https://doi.org/10.1039/C6TA00890A. 